\title{
Digital Predistorter in Crosstalk Compensation of MIMO Transmitters
}

\author{
Sara Hesami, John Dooley, Ziming, Wang, and Ronan Farrell \\ Electronic Engineering. National University of Ireland Maynooth (NUIM) \\ Maynooth, Ireland \\ sara.hesami@nuim.ie
}

\begin{abstract}
Due to the proximity of transmit paths Multipleinput multiple-output (MIMO) systems are vulnerable to crosstalk effects. This paper presents a comparison between direct learning architecture (DLA) and indirect learning architecture (ILA) for cancellation of crosstalk through digital pre-distortion. A theoretical background detailing the reasons for the improved performance of the DLA method is given. Measured results are presented showing the benefits of the DLA estimation method compared to the ILA estimation method in the presence of antenna crosstalk.
\end{abstract}

Keywords-Digital predistortion; multiple input multiple output (MIMO); linear crosstalk; antenna crosstalk; direct learning; power amplifiers.

\section{INTRODUCTION}

Increasing demand for high capacity and high data-rate poses major challenges for the wireless networks in $5 \mathrm{G}$. Massive multiple-input multiple-output (MIMO) wireless systems have been proposed as one solution to meet the requirements of a 1-Gb/s transmission rate [1] and small cell deployments to increase the capacity. Compact design of radio base stations (RBS) is essential to the development of small cell together with massive MIMO implementations. In this regard, the RBS needs to be developed using single chipsets as much as possible. However, MIMO wireless systems are vulnerable to the effect of crosstalk when implemented on a single chipset [2].

In MIMO systems, crosstalk mainly occurs due to coupling effects of signals between the transmission branches [2]. Two main types of crosstalk have been identified based on whether the crosstalk occurs before or after the power amplifiers (PA). Crosstalk which occurs before the PAs is subject to the nonlinearity of the PA and therefore is called nonlinear crosstalk. In this paper, we will focus on the crosstalk which occurs after the PAs, which is known as linear or antenna crosstalk [3]. The fundamental issue with antenna crosstalk is that the distortion caused by this effect occurs in-band.

A number of crosstalk cancelation techniques for chip design have been proposed [4] at the cost of current consumption. In other cases the crosstalk is avoided by spacing the channels, isolation between the physical signal paths and differential I/Os which can be used at the expense of increased power consumption and lower data rate [4]. All of these solutions mentioned above also introduce added HW design complexity. It is therefore advantageous if it is possible to compensate for crosstalk effects in the digital hardware already used in the RBS. For this reason, in high power MIMO base stations crosstalk cancelation using digital predistortion (DPD) has become popular. DPD is an essential unit in an RBS to cancel the nonlinearity of high power amplifiers. Crosstalk can be treated as a source of nonlinearity and the existing DPD stage can therefore be adapted to compensate for its effects also.

The memory polynomial based model is widely used for single-input single-out (SISO) PAs. The generalized memory polynomial (GMP) behavioral model introduced in [5], shows excellent accuracy at the cost of additional complexity [6]. A SISO GMP model was adapted in [7] to compensate the linear and nonlinear crosstalk effects in $2 \times 2$ MIMO system. The adapted behavioral model for antenna crosstalk was named generalized memory polynomial for linear crosstalk (GMPLC). A similar model to the GMP is the memory polynomial (MP). In this paper a memory polynomial is used to model the distortion of a set of nonlinear PAs combined with antenna crosstalk effects. The memory polynomial can achieve comparable accuracy, using fewer coefficients compared to the GMP approach. In this way a more computationally efficient approach can be achieved. This is directly beneficial in both the case of modelling MIMO and in particular M-MIMO systems. The same benefits for the MP structure used to model these systems will also apply in its application to DPD.

In calculating the DPD coefficients, there are two main strategies which can be employed, namely direct or indirect learning. Previous studies such as [7] [12], have used the indirect learning architecture to calculate DPD coefficients for a set of nonlinear PAs with crosstalk. Recent studies have shown that direct learning has superior advantages over the indirect learning architecture when applied to DPD [8] [9]. Direct learning performs better in the presence of noise and in particular when applied to compensation of crosstalk effects.

In Section II the theoretical background to antenna crosstalk is introduced. Then, PA behavioral modeling with crosstalk is reviewed and finally an inverse control technique used in this study is explained. 


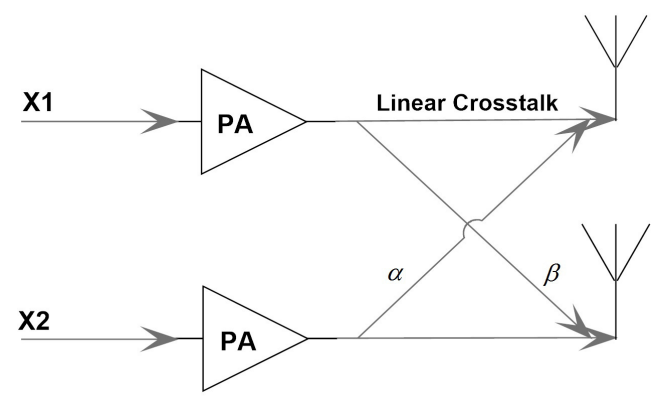

Fig. 1. Antenna Crosstalk in a $2 \times 2$ MIMO Transmitter

In Section III the implementation of direct learning architecture in crosstalk behavioral modeling is introduced and measured results are presented.

\section{MEthodology}

\section{A. Antenna Crosstalk}

Antenna crosstalk or linear crosstalk is the effect of signal coupling which occurs after the PA in the transmission line. Because the crosstalk terms after the PA do not pass through a nonlinear component in the RF front-end, this type of coupling effect is considered antenna crosstalk. As a result, the impairment effects will appear as in-band distortion [2] and as a result will increase the measured error vector magnitude (EVM) of the signal which is fed to the antenna.

In Fig. 1 the mechanism by which antenna crosstalk occurs is shown. It can be seen that some proportion of the signal in each path is transferred to the adjacent signal path. Not only will there be antenna crosstalk between the transmitter paths, but there is also crosstalk in the channel and crosstalk between the receiver antennas. Compensation of all of these crosstalk effects can be compensated for in the receiver, however if the transmitter antenna crosstalk can be compensated prior to signal transmission the overall performance of the MIMO system will be improved [11]. Considering this fact, it is advantageous to compensate the linear or antenna crosstalk in the transmitter DPD prior to transmission.

The antenna crosstalk model presented in [2] and [7] is as follows

$$
\begin{aligned}
& y_{1}(n)=f_{1}\left(x_{1}(n)\right)+\alpha * f_{2}\left(x_{2}(n)\right) \\
& y_{2}(n)=\beta^{*} f_{1}\left(x_{1}(n)\right)+f_{2}\left(x_{2}(n)\right)
\end{aligned}
$$

$y_{1}(n)$ and $y_{2}(n)$ are the outputs of the antennas in branches one and two respectively in a $2 \times 2$ MIMO system. $x_{1}(n)$ and $x_{2}(n)$ are the inputs to the PA in branch one and two respectively. While, $\alpha$ and $\beta$ are the crosstalk factors as shown in Fig. 1. In this regard $f_{1}$ is a function representing the nonlinearity effects in branch one and $f_{2}$ is the function representing the nonlinearity effects in branch two. By modeling the crosstalk effect in this way, the output of the antenna will be a linear combination of the nonlinear effects from its reference branch and a factor of nonlinear effects from the second branch.

Accordingly, no cross terms are needed to model the coupling effect of antenna crosstalk. The nonlinearities are modeled separately therefore, any conventional SISO model can be used to replace each of $f_{1}$ and $f_{2}$ functions.

\section{B. Behavioral Modeling and DPD with the Antenna Crosstalk}

Exemplified in a number of studies, the MP model has been extensively applied to the digital pre-distortion of RF power amplifiers. It can be argued that the relative accuracy of MP is lower than other methods such as full Volterra (FV), GMP, second order dynamic deviation reduction (DDR), direct dynamic artificial neural network (DD-ANN) and recursive dynamic ANN (RD-ANN) models. However, its computational simplicity in comparison to these other approaches makes it far more attractive for certain applications. The complex baseband representation of the MP model achieved in [10] is

$$
y(n)=\sum_{k=1}^{K} \sum_{q=0}^{Q} a_{k q} x(n-q)|x(n-q)|^{k-1}
$$

With input $x(n)$ and output $y(n)$ and the predistorter has the memory depth of $Q$ and maximum nonlinearity of $K$. As earlier discussed in Section I.A this model can be extended and used in a antenna crosstalk model of (1) which results in

$$
\begin{aligned}
& y_{1}(n)=\sum_{k=1}^{K} \sum_{q=0}^{Q} a_{1 k q} x_{1}(n-q)\left|x_{1}(n-q)\right|^{k-1} \\
& +\sum_{k=1}^{K} \sum_{q=0}^{Q} a_{2 k q} x_{2}(n-q)\left|x_{2}(n-q)\right|^{k-1}
\end{aligned}
$$

The effect of $\alpha$ is reflected in coefficients $a_{1 k q}$ and $a_{2 k q}$ and does not need to be estimated separately [7]. Later this model will be validated for pre-distorter parameter estimation in Section III.

\section{Direct Learning and Indirect Learning Architecture}

Indirect Learning architecture (ILA) and direct learning architecture (DLA) are two widely used methods for DPD coefficient estimation. Considering Fig. 2, the normalized input to the DPD, $x(n)$ and the normalized output from PA $y(n)$ and the error is mentioned in [8] as

$$
\varepsilon(n)=y(n)-x(n)
$$




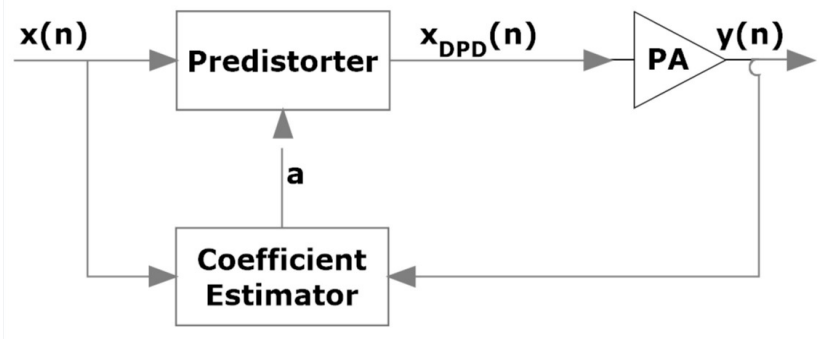

Fig. 2. Direct Learning Architecture

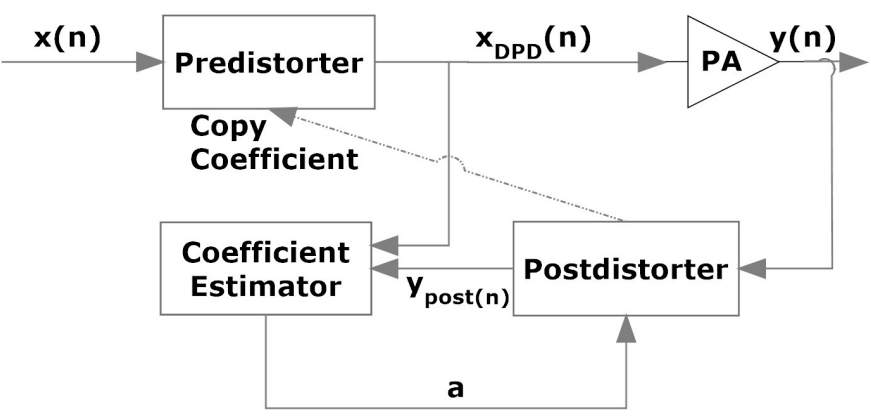

Fig. 3. Indirect Learning Architecture

In DLA, the DPD block is located within the estimation loop as shown in Fig. 2. The cost function to be minimized [8] is

$$
J_{D L A}=E\left[\left|\mathcal{E}(n)-\psi_{x}(n) . \Delta a\right|^{2}\right]
$$

The $\psi_{x}(n)$ represent the basis function based on $x(n)$ and its previous values. In each iteration the coefficient estimation is performed based on the calculation of the error in coefficient estimation, $\Delta a$. Referring to (2) the basis function is built only with respect to the input $x(n)$ and its previous values.

In ILA, a post-distorter is applied to the normalized $y(n)$ and the aim is to minimize the difference between predistorted $x_{D P D}(n)$ and the post-distorted $y_{\text {post }}(n)$, as shown in Fig. 3. This results in calculating the coefficients used in the calculation of $y_{\text {post }}(n)$. The cost function introduced in [8] aims to minimize the error between the pre-distorter output and the post distorted output signals as given in (6).

$$
J_{I L A}=E\left[\left|x_{D P D}(n)-y_{\text {post }}(n)\right|^{2}\right]
$$

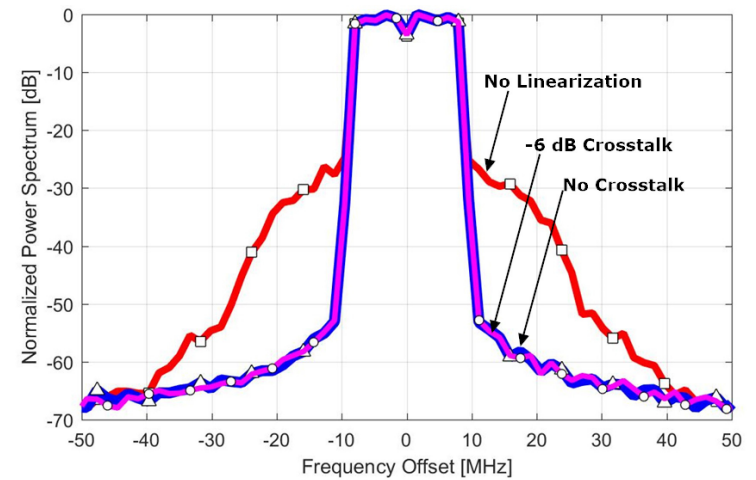

Fig. 4. PA output spectrum when no linearization was applied, PA output with linearization in presence of no crosstalk and -6dB crosstalk

Looking again in the polynomial (2) in ILA the basis function to be arranged with respect to the $y(n)$ to obtain the $y_{\text {post }}(n)$.

Relying on $y_{\text {post }}(n)$ in the coefficient estimation is a primary source of instability in using the ILA method. This is as a result of the uncertainty in the estimated values for $y_{\text {post }}(n)$ and $x_{D P D}(n)$, which are in turn used to approximate the coefficient values. The DLA method directly uses the input and output signals in the approximation of the coefficients. In the presence of antenna crosstalk the output signal $y(n)$ will be distorted by the coupling effect from the adjacent branch PA output. This additional component will serve to create even more uncertainty in the estimation of the signal $y_{\text {post }}(n)$.

Later we see in simulation and results that the performance of MPLC by means of DLA gives better EVM compare to the ILA which is more vulnerable to any noise in $y(n)$ measurements.

\section{MEASURED RESULTS}

In order to compare the performance of DLA and ILA coefficient estimation in the presence of antenna crosstalk a simulation is carried out over a $2 \times 2$ MIMO model where two branches have two identical PAs. An LTE single carrier signal with the bandwidth of $20 \mathrm{MHz}$ is used with the number of samples for each signal sets to 100,000 samples and the signals sampled by the sampling rate of $f_{s}=208 \mathrm{MHz}$. An additive white Gaussian noise is added at the output of the PA which leads to a signal to noise ratio (SNR) of $65 \mathrm{~dB}$.

In the MPLC model (3) the order of nonlinearity set to $K=$ 4 and memory depth set to $Q=9$. For the above signals, this model gives a normalized mean-square error (NMSE) equal to $-48 \mathrm{~dB}$ and an adjacent channel power ratio (ACPR) of -57.73 $\mathrm{dB}$. 
TABLE I. EVM [\%] FOR DIFFERENT ANTENNA CROSSTALK IN A $2 \times 2$ MIMO TRANSMITTER USING DLA AND ILA COEFFICIENT ESTIMATION TECHNIQUES

\begin{tabular}{|l|l|c|c|}
\hline & No Crosstalk & $\begin{array}{c}-10 \text { dB Antenna } \\
\text { crosstalk }\end{array}$ & $\begin{array}{c}-6 \text { dB Antenna } \\
\text { crosstalk }\end{array}$ \\
\hline DLA & 0.35 & 0.35 & 0.38 \\
\hline ILA & 0.37 & 0.42 & 0.60 \\
\hline
\end{tabular}

Fig. 4 shows the spectrum of PA output without any linearization technique be implemented. Then the spectrum of PA output with linearization technique presented when $a$ ) there is no crosstalk and $b$ ) in presence of $-6 \mathrm{~dB}$ antenna crosstalk.

When the linearization technique is used, the out-of-band characteristics of spectrum in case $a$ and $b$ are closely similar.

To compare the relative performance of the ILA and DLA, antenna crosstalk figures of $-6 \mathrm{~dB}$ and $-10 \mathrm{~dB}$ are considered in this work. In Table. I, the EVM provided in the presence of no crosstalk, $-6 \mathrm{~dB}$ antenna crosstalk and $-10 \mathrm{~dB}$ antenna crosstalk when the DLA and ILA used for DPD coefficient estimation. In the absence of antenna crosstalk, the EVM obtained using the ILA method which is comparable to that achieved using the DLA method.

By adding the effect of crosstalk, the DLA method maintained the same EVM performance which it achieved without crosstalk. However, repeating the same procedure for the ILA method the EVM increased considerably, first to 0.42 $\%$ and then to $0.61 \%$ for $-6 \mathrm{~dB}$ and $-10 \mathrm{~dB}$ crosstalk. In all three cases by using the DLA method to estimate the DPD coefficients a lower EVM was obtained.

As discussed in Section II. $C$, the ILA method uses two signals which are themselves estimations of the desired predistorted signal and post-distorted signals. DLA on the other hand uses the original input signal and the measured output signal directly to calculate the pre-distorter coefficients.

In Fig. 5 a random selection of MPLC DPD coefficients are presented for both DLA and ILA methods in the presence of different amounts of antenna crosstalk. It can be seen from Fig. 5 by the separation of the lines that the coefficients calculated with the ILA method fluctuate more than those calculated using the DLA method. These observations closely resemble the improved EVM performance of DLA in comparison to ILA.

\section{CONCLUSION}

In this paper, two different estimation methods were evaluated for the calculation of DPD coefficients for systems that include crosstalk. These methods were applied to a antenna crosstalk cancelation of a $2 \times 2$ MIMO transmitter. The MPLC was used to model the nonlinearity in the transmitter and used in the DPD implementation. EVM is used to present the relative performance of the two methods. The measured results demonstrate the improved performance of

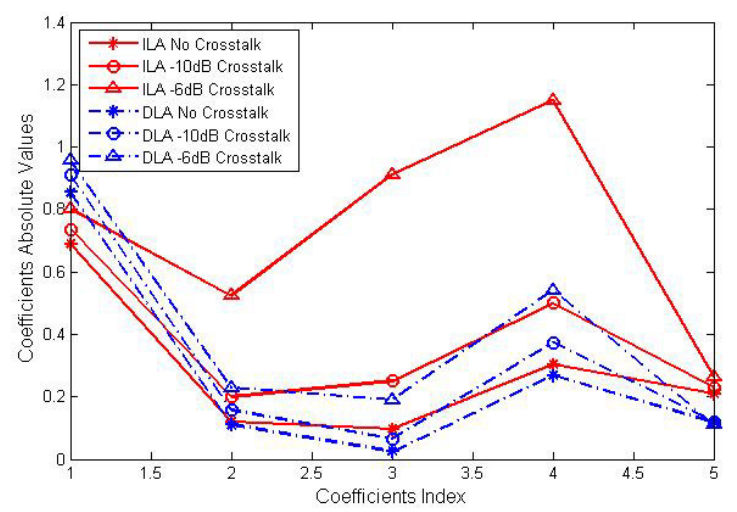

Fig. 5. Coefficients Divergence in DLA and ILA in presence of no crosstalk, $-10 \mathrm{~dB}$ antenna crosstalk and $-6 \mathrm{~dB}$ antenna crosstalk

DLA estimation in comparison to ILA estimation. In the presence of crosstalk the DLA method can maintain good EVM performance. In contrast to the ILA method where it can be seen the EVM performance degardes considerably in the presence of antenna crosstalk.

\section{Acknowledgment}

This publication has emanated from research supported in part by a research grant from Science Foundation Ireland (SFI) and is co-funded under the European Regional Development Fund under Grant Number 13/RC/2077.

\section{References}

[1] Paulraj, A.J.; GORE, D.A.; Nabar, R.U.; Bolcskei, H., "An overview of MIMO communications - a key to gigabit wireless," in Proceedings of the IEEE, vol.92, no.2, pp.198-218, Feb 2004.

[2] S. Bassam, M. Helaoui, and F. Ghannouchi, "Crossover digital predistorter for the compensation of crosstalk and nonlinearity in MIMO transmitters," IEEE Trans. Microw. Theory Techn, vol. 57, no. 5, pp. 1119-1128, May 2009.

[3] Saffar, D.; Boulejfen, N.; Ghannouchi, F.M.; Gharsallah, A.; Helaoui, M., "Behavioral Modeling of MIMO Nonlinear Systems With Multivariable Polynomials," in Microwave Theory and Techniques, IEEE Transactions on , vol.59, no.11, pp.2994-3003, Nov. 2011.

[4] Taehyoun Oh; Harjani, R., "A 6-Gb/s MIMO Crosstalk Cancellation Scheme for High-Speed I/Os," in Solid-State Circuits, IEEE Journal of, vol.46, no.8, pp.1843-1856, Aug. 2011.

[5] Morgan, D.R.; Zhengxiang Ma; Jaehyeong Kim; Zierdt, M.G.; Pastalan, J., "A Generalized Memory Polynomial Model for Digital Predistortion of RF Power Amplifiers," in Signal Processing, IEEE Transactions on, vol.54, no.10, pp.3852-3860, Oct. 2006.

[6] Lei Guan; Anding Zhu, "Green Communications: Digital Predistortion for Wideband RF Power Amplifiers," in Microwave Magazine, IEEE, vol.15, no.7, pp.84-99, Nov.-Dec. 2014.

[7] Amin, S.; Landin, P.N.; Handel, P.; Rönnow, D., "Behavioral Modeling and Linearization of Crosstalk and Memory Effects in RF MIMO Transmitters," in Microwave Theory and Techniques, IEEE Transactions on, vol.62, no.4, pp.810-823, April 2014.

[8] Braithwaite, R.N., "A comparison of indirect learning and closed loop estimators used in digital predistortion of power amplifiers," 
in Microwave Symposium (IMS), 2015 IEEE MTT-S International, vol., no., pp.1-4, 17-22 May 2015.

[9] Paaso, H.; Mammela, A., "Comparison of direct learning and indirect learning predistortion architectures," in Wireless Communication Systems. 2008. ISWCS '08. IEEE International Symposium on, vol., no., pp.309-313, 21-24 Oct. 2008.

[10] Ding, Lei; Zhou, G.T.; Morgan, D.R.; Zhengxiang Ma; Kenney, J.S.; Jaehyeong Kim; Giardina, C.R., "A robust digital baseband predistorter constructed using memory polynomials," in Communications, IEEE Transactions on, vol.52, no.1, pp.159-165, Jan. 2004.
[11] Proakis, J.G. (2005) Digital Communications, McGraw Hill, New York.

[12] Y. J. Liu, W. Chen, J. Zhou, B. H. Zhou and F. M. Ghannouchi, "Digital Predistortion for Concurrent Dual-Band Transmitters Using 2-D Modified Memory Polynomials," in IEEE Transactions on Microwave Theory and Techniques, vol. 61, no. 1, pp. 281-290, Jan. 2013. 